Chapter 4

\title{
Role of Oxidative Stress in A $\beta$ \\ Animal Model of Alzheimer's Disease: \\ Vicious Circle of Apoptosis, \\ Nitric Oxide and Age
}

\author{
Ferihan Cetin
}

Additional information is available at the end of the chapter

http://dx.doi.org/10.5772/54718

\section{Introduction}

The aging process is believed to be closely related to increased oxidative stress. Reactive intermediates of oxidative stress affect the cellular redox status and induce apoptosis [1]. Oxidative stress due to the loss of balance between ROS production and antioxidant defenses affects all the vital organs, resulting in aging $[1,2]$. Oxidative damage, mitochondrial dysfunction and inflammation underlies many common aging-related neurodegenerative diseases, including $\mathrm{AD}[2,3]$. The major pathological hallmark of $\mathrm{AD}$ is the accumulation of $\mathrm{A} \beta$ peptides in the brain [4]. Oxidative insults that induce neuronal apoptosis, including agents that induce membrane lipid peroxidation, also have been shown to activate caspases [5]. Increased lipid peroxidation was consistently observed in some animal models of Alzheimer amyloidosis [4, 6]. It has been shown that a single $A \beta$ administration into the rat hippocampus could induce increase of NOS activity and NO level [6]. Nitric oxide is a multifunctional molecule that acts as messenger/modulator in synaptogenesis and potential neurotoxin and is synthesized by three isozymes of Nitric oxide synthase (NOS) [7]. Oxidative stress reflects a situation in which ROS is continuously produced and exceeds the capacity of endogenous antioxidant defense systems. Several studies have suggested that oxidative stress plays a key role in A $\beta$-mediated neuronal cytotoxicity by triggering or facilitating neurodegeneration through a wide range of molecular events that eventually lead to neuronal cell loss. A $\beta$ significantly increases production and enhances membrane lipid peroxidation, leading to neuronal apoptosis [8,9]. Because multiple factors are involved in the pathogenesis of the AD, it is difficult to find an ideal in vivo model. It is important to determine $A \beta$ 1-42 injection effects especially in hippocampus, 
temporal and parietal cortex, because a definite atrophy reveals in these regions in the late stage of sporadic AD. The fact that revealing changes with age are the accumulation of ROS and the onset of apoptosis cascade, these interfering subjects have been investigated intensely nearly for two decades. $\beta$-amyloid itself is a source of free radicals. One possible mechanism for initiating apoptosis could be the generation of free radicals by the peptide leading to lipid peroxidation and oxidative stress $[9,10]$. Many recent studies have confirmed the toxicity of peroxynitrite to neurons and the involvement of nitric oxide in neurologic pathologies $[10,11]$. Other suspects include the superoxide radical hydrogen peroxide, which is implicated in amyloid neurotoxicity, and peroxynitrite, which can be formed by combining superoxide and nitric oxide [9].

Nitric oxide is a multifunctional molecule that acts as messenger/modulator in synaptogenesis and potential neurotoxin and is synthesized by three isozymes of NOS. Entorhinal cortex neurons are highly vulnerable to neurodegeneration in AD express low levels of NOS and the inducible form of NOS is upregulated in AD [14]. They found a significant decrease in the number of cells expressing detectable levels of nNOS mRNA in the white matter underlying the frontal cortex and in the dentate gyrus and CA subfields of the hippocampus in AD. Furthermore, there was also a significant decrease in the number of NADPHd-positive cells in the dentate gyrus and CA subfields of the hippocampus in AD [15].

It has been suggested that the upregulation of nNOS and subsequent $\mathrm{NO}$ release in certain classes of cortical interneurons may be one of the earliest events signaling the apoptosis of cortical projection neurons in lesion models [16]. Conflicts may arise from varying emphasis on different cortical areas among these investigators and the complex anatomy of cortical nitrinergic neurons that comprise at least three distinct cell populations, that is, large subcortical whiter matter neurons, large nNOS (+) interneurons, and smaller nNOS (+) interneurons that are often missed because of low levels of nNOS/NADPHd expression [16]. Not only neurons but also activated microglia are capable of releasing neurotoxic molecules, such as proinflammatory cytokines and toxic oxygen and nitrogen species [17]. The relationship between mitochondrial damage, glutathione status/GSH dependent enzymes, oxidative stress, and neuronal dysfunction has been demonstrated by the effects of excessive production of $\mathrm{H}_{2} \mathrm{O}_{2}$ within mitochondria, which leads to depletion of mitochondrial GSH. Besides, measurements of the activity time course of antioxidant enzymes are important when comparing the alterations induced by $\mathrm{A} \beta$ with those found in $\mathrm{AD}$ patients [18]. Glutathione is known to protect cells against apoptosis, which is consistent with the suggestion of involvement of $\mathrm{ONOO}^{-}$mediated cellular events in neuronal apoptosis $[2,17]$.The current literature contains contradictory results about the dual role (neuroprotector/neurotoxic) that NO may play in the aging CNS [19]. It is mostly believed that NO has neurotoxic effects on neurons [20,21]. It is now known that, in aging and Alzheimer brain, nNOS-expressing hippocampal neurons are more vulnerable to oxidative stress [14]. A $\beta(25-35)$ activated nNOS in the cerebral cortex and hippocampus without effect on iNOS activity [6]. Since A $\beta$ neurotoxicity is believed to be mediated by $\mathrm{NO}$ and the potential toxic effects of $\mathrm{NO}$ depend on the intracellular source of the molecule (i.e. isoform-specific) [7]. 


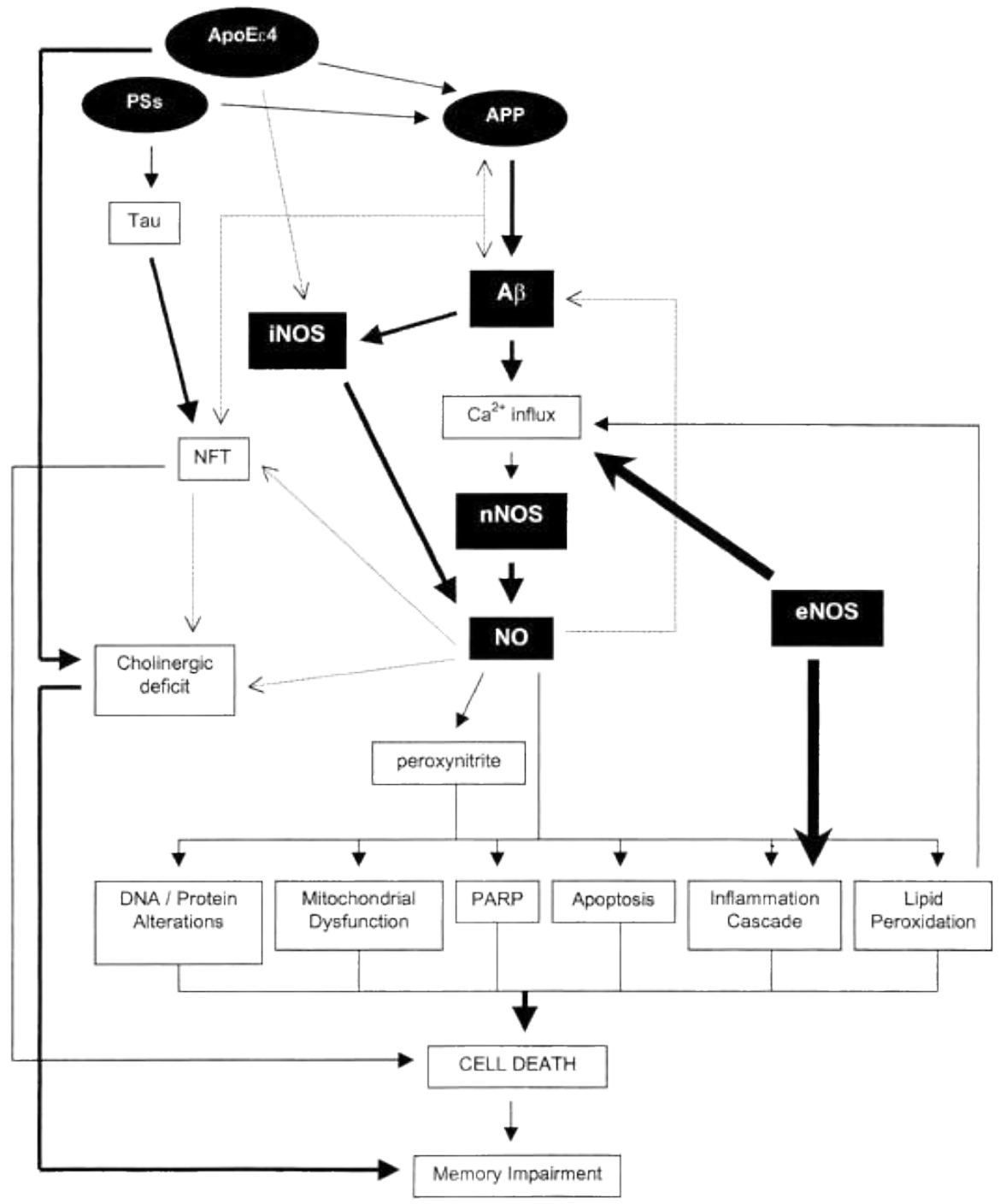

Figure 1. Nitric oxide (NO) neurotoxicity and neuroprotectivity in relation to Alzheimer's disease (AD). Mutations of presenilins (PSs) and amyloid precursor protein (APP) are associated with increased production of A $\beta$. Neurofibrillary tangles (NFT) formation is the result of tau hyperphosphorylation, which leads to cell death secondary to cellular trafficking disruption. PSs have also been implicated in the process of tau hyperphosphorylation. Apolipoprotein E e4 (ApoE e4) genotype is considered a risk factor for AD. It appears to affect A $\beta$ production and a correlation between ApoE e4 and cholinergic deficit has been established. Cholinergic deficit is one of the most significant findings in AD, and is implicated in memory impairments observed in this disease. Increased production of A $\beta$ induces NO production either by disrupting Ca homeostasis and subsequent increased in intracellular Ca (nNOS and eNOS-mediated NO release) or by interactions with glial cells (iNOS-mediated NO release). NO is a free radical and can produce peroxynitrite. These reactive oxygen species induce a variety of neurotoxic mechanisms, including DNA/ protein alterations, mito- 
chondrial dysfunction, poly ADP-ribose polymerase (PARP) overactivation, apoptosis, neuro-inflammation, and lipid peroxidation (which jeopardize cellular membrane integrity, which leads to further Ca influx and NO release). These mechanisms are likely to be involved in cell death and memory impairments observed in AD. Several potential relationships may exist between various AD markers (dashed arrows). ApoE e4 may induce iNOS-mediated NO production. NFT formation may influence $A \beta$ accumulation and vice versa, and that APP metabolism may play a role in tau phosphorylation and subsequent NFT formation. NO, by activating a variety of signalling molecules, may induce $A \beta$ and NFT formation. In ischemia, eNOS-mediated NO production appears to be neuroprotective by decreasing Ca influx and neuro-inflammation (thickened arrows). This NOS isoform may have a similar role in neurodegenerative diseases such as AD. [Law A., Gauthier S., Quiron R., Say NO to Alzheimer's disease: putative links between nitric oxide and dementia of the Alzheimer's type. 2001, ref 7]

\section{2. $\beta$-amyloid peptide in Alzheimer's disease}

In 1911, Alois Alzheimer described a neuropsychiatric disorder affecting the elderly, which is widely known today as Alzheimer's disease (AD). Early studies of patients afflicted with this disease demonstrated, via silver staining, the presence of lesions in the brain cortex. Those lesions corresponded to neurofibrillary tangles (NFTs), which are histopathologic structures localized within the neuronal cells. The majority of cases of AD correspond to the sporadic form of this disorder. Approximately $5-10 \%$ of patients present an autosomal mode of transmission and account for cases called familial Alzheimer's disease (FAD). The role of amyloid deposits in brain as a triggering factor for Alzheimer's disease has obtained increasing scientific support since Glenner's discovery in 1984. The amyloid precursor protein (APP) belongs to a type 1 transmembrane family of glycoproteins that is ubiquitously expressed in several types of cells. The N-terminal moiety of APP is projected toward the extracellular domain or can be localized in the lumen of intracellular vesicles, such as those of the endoplasmic reticulum, Golgi apparatus, and intracellular endosomes. On the other hand, the APP C-terminal region lies in the cytoplasmic domain. APP is sensitive to proteolysis by a set of proteases called $\alpha, \beta, \gamma$ secretases. Secretases are responsible for the production of $A \beta(1-40)$ peptide or the $A \beta(1-42)$ variant with a significantly higher capacity to self-aggregate.[22]

\section{A $\beta$ animal models}

A critical question that must be addressed in examining any animal model of a human disease is how well the animal model mimics the mechanisms and ultimate pathology observed in the human disease. [23] Clearly, there are differences between the present rat model and human AD. First, whereas in a study gliosis and pathology are observed over a time course of $30 \mathrm{~d}$, in human AD the time course is on the order of decades. Second, the amyloid load per unit of brain area is generally higher and the amyloid distribution more extensive in the human AD brain than in their rat model. However, a key difficulty in addressing the mechanisms of pathology in human AD is that one rarely looks at human brain tissue at the initiation of the disease but rather examines the $\mathrm{AD}$ brain at the end stage of the disease, long after the initial mechanisms of pathology occur. In behalf of these discrepancies intracerebral or intracerebroventricular $A \beta$ injections or infusions are used to mimic $A D .[23,24]$ 
Thus, the results strongly suggest that injection of fibrillar $\mathrm{A} \beta$ activates both microglia and astrocytes. Although both microglia and astrocytes show a dramatic upregulation of iNOS expression in response to injection of $\mathrm{fA} \beta$, there are marked spatial and morphological differences in the microglia and astrocyte responses to $\mathrm{fA} \beta$. Whereas microglia surround and phagocytize $\mathrm{fA} \beta$, astrocytes show no evidence of $A \beta$ phagocytosis but rather form a virtual wall between microglia containing fA $\beta$ and the surrounding neurons.[23] The fA $\beta$ induces neuronal loss and a significant increase in iNOS expression by microglia and astrocytes in vivo, suggesting that it is the release of bioactive molecules like nitric oxide by microglia and astrocytes, rather than direct contact between $A \beta$ fibrils and neurons, that mediates $A \beta$ neurotoxicity in AD.[23]

\section{A $\beta$ and oxidative stress}

In recent years, considerable data have accrued indicating that the brain in $\mathrm{AD}$ is under increased oxidative stress and this may have a role in the pathogenesis of neuron degeneration and death in this disorder. The direct evidence supporting increased oxidative stress in AD is: (1) increased brain $\mathrm{Fe}, \mathrm{Al}$, and $\mathrm{Hg}$ in $\mathrm{AD}$, capable of stimulating free radical generation; (2) increased lipid peroxidation and decreased polyunsaturated fatty acids in the AD brain, and increased 4-hydroxynonenal, an aldehyde product of lipid peroxidation in AD ventricular fluid; (3) increased protein and DNA oxidation in the AD brain; (4) diminished energy metabolism and decreased cytochrome c oxidase in the brain in AD; (5) advanced glycation end products (AGE), malondialdehyde, carbonyls, peroxynitrite, heme oxygenase- 1 and SOD-1 in neurofibrillary tangles and AGE, heme oxygenase-1, SOD-1 in senile plaques; and (6) amyloid beta peptide is capable of generating free radicals. [25]

Oxidative insults emanating from within the cell can threaten homeostasis if they are not appropriately resolved. Mitochondria actively and continuously generate ROS during respiration, favoring a situation of mitochondrial oxidative stress. The electron transport chain is an essential mechanism for generation of cellular energy and is localized to the mitochondrial inner membrane. Autooxidation of reduced respiratory chain components cause the production of free radical intermediates, $\mathrm{O}_{2}$ and $\mathrm{H}_{2} \mathrm{O}_{2}$, which in the presence of iron can produce hydroxyl radical $(\bullet \mathrm{OH})$. There are two specific sites where electrons may leak out of the chain to partially reduce oxygen. One is the NADH dehydrogenase and the other is at the ubiquinone cytochrome $b$ intersection. These oxygen species are dealt with by superoxide dismutases (SOD), enzymes that are considered to be the first line of defense against oxygen toxicity, and exist in two forms in mammalian tissues: copper, zinc (Cu, Zn SOD), and manganese (Mn SOD) metalloproteins. Although mitochondria are notorious for ROS production, they are not the only sites of intracellular oxidative stress. In the cytosol, the arachadonic acid cascade, yielding prostaglandins, and leukotrienes may generate ROS when the released lipid is metabolized, and some cytochrome $\mathrm{P}-450$ isozymes are notorious $\mathrm{O}_{2}^{-2}$ producers. Several possibilities have been proposed for the mechanisms of NO-mediated cytotoxicity. [26] First, formation of ironnitrosyl complexes with critical FeScontaining enzymes (e.g., aconitase), may cause an impairment of mitochondrial function and energy depletion. Second, $\mathrm{NO}^{-}$may directly 
damage chromatin by deamination and cross-linking of DNA, which increases mutagenesis. Third, generation of peroxynitrite by a reaction between $\mathrm{NO}^{-}$and superoxide $\left(\mathrm{O}_{2}^{-2}\right)$ may play a significant role in the cytotoxic process. Fourth, $\mathrm{NO}^{-}$may inactivate several antioxidant enzymes, including catalase, glutathione peroxidase, and superoxide dismutases. Also, $\mathrm{NO}^{-}$ has been reported to induce apoptosis by increasing ceramide generation through caspase-3 activation, induction of mitochondrial permeabilittransition, and activation of the Fas system. The mechanism of action of many exogenous agents involves redox cycling whereby an electron is accepted to form a free radical and it is then transferred to oxygen. [26]

Although NO has many important and beneficial physiologic functions, it also can play a role in neurodegenerative disease pathology. In these diseases, NO is produced in excess by iNOS induction owing to the proinflammatory response, which is a common feature of neurodegenerative disorders. Moreover, NO is much more harmful under pathologic conditions that involve the production of reactive oxygen species (ROS), such as superoxide anion and the formation of peroxynitrite. Two important properties of NO that may contribute to its pathologic functions are its ability to modify proteins through nitrosylation and nitrotyrosination and its ability to react with oxygen to form RNS. [27]

In mature neurons, when cytosolic nNOS is the primary producer of $\mathrm{NO}, \mathrm{Ca}^{2+}$ entry through overactive NMDA channels stimulates nNOS; thus, NO can then enter the mitochondria, directly inhibiting complex IV (cytochrome c oxidase) of the respiratory chain, which leads to a block of ATP production and eventual cell death due to energetic failure. [27]

Furthermore, NO has been shown to activate both the constitutive and inducible isoforms of cyclooxygenase, which are upregulated in brain cells under proinflammatory conditions. During the catalytic cycle of cyclooxygenase, the release of free radicals and the formation of prostaglandins occur, two events closely related to the development of neuroinflammation. [27]

Hsp90 is another protein associated with AD that undergoes nitrosylation. S-Nitrosylation of Hsp90 abolishes ATPase activity that is necessary for its chaperone function; thus, inactivation of Hsp90 may allow accumulation of tau and A $\beta$ aggregates in the AD brain. It is generally recognized that mitochondria continuously undergo two opposing processes, fission and fusion. The disruption of this dynamic equilibrium may herald cell injury or death and may contribute to developmental and neurodegenerative disorders. Nitric oxide produced in response to $\beta$-amyloid protein has been shown to trigger mitochondrial fission, synaptic loss, and neuronal damage, in part through S-nitrosylation of dynamin-related protein. [27]

Many studies showed amyloid-beta interaction with different receptors in the cellular membrane of the vasculature, neurons, oligodendrocytes, and glial cells where it is transported from cell surface into endosomal and lysosomal compartments. The aberrant signalling of these receptors in AD triggered an abnormal accumulation of amyloid-beta into cytosolinducing cellular stress underlies to neuronal dysfunction and dementia. It is described that these receptors can be megalin, also known as low-density lipoprotein related protein-2 (LRP2), LRP-1, or RAGE (receptors for advanced glycation end products). The interaction of these receptors with amyloid-beta in neurons, microglia, and vascular cells accelerates and amplifies deleterious effects on neuronal and synaptic functions. [28] These findings are further 
in line with the recently proposed hypothesis of an intracellular amyloid-beta toxicity cascade which suggests that the toxic amyloid-beta species intervening in molecular and biochemical abnormalities may be intracellular soluble aggregates instead of extracellular, insoluble plaques. There are many studies proposing that megalin- and/or RAGE-dependent signalling are involved in the regulation of amyloid-beta clearance and probably may contribute to amyloid pathology and cognitive dysfunction observed in the AD patients and AD mouse model. [28]

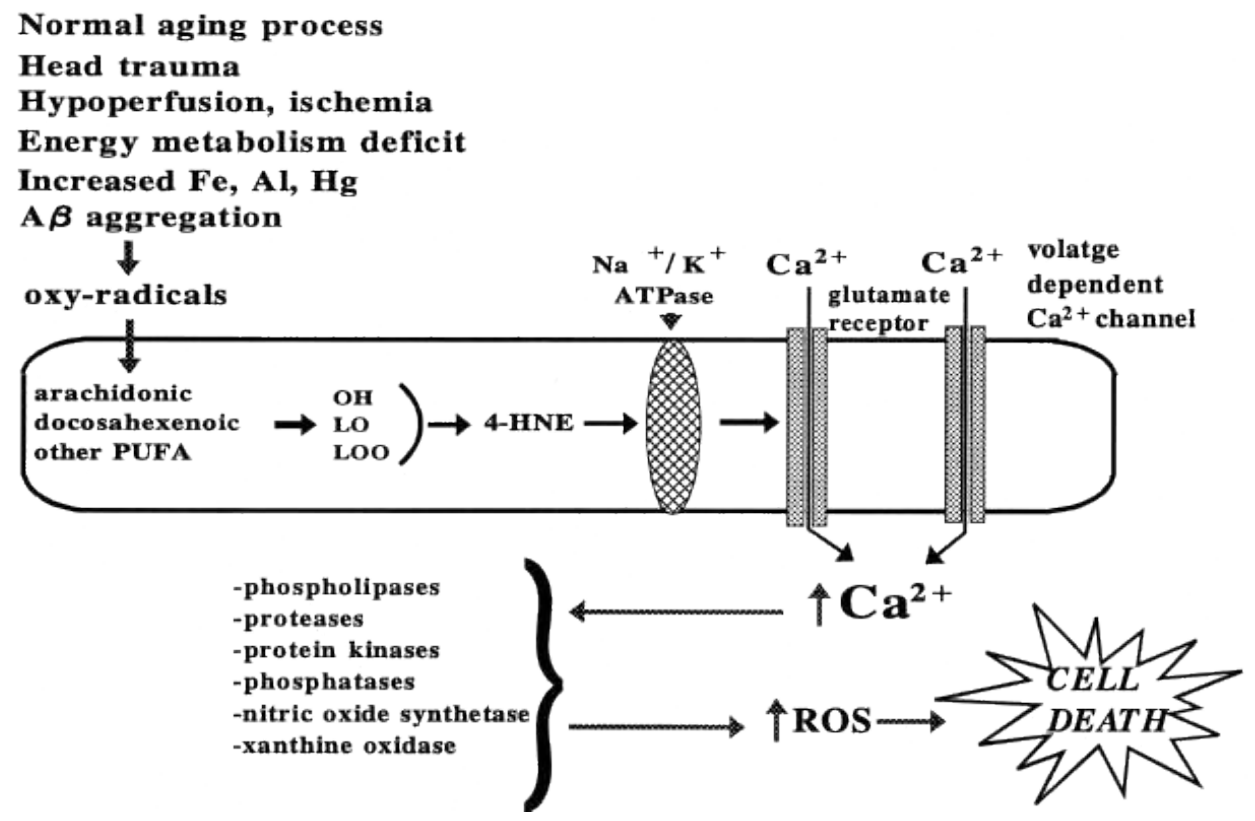

Figure 2. Mechanism of potential neuron death in AD. Anumber of conditions may cause free radical generation that can lead to peroxidation of membrane polyunsaturated fatty acids. This leads to formation of other oxygen radicals and generates aldehydes such as 4-hydroxynonenal (HNE). 4-HNE is capable of altering membrane ATPases leading to increased intracellular calcium, which initiates a cascade of events leading to further free radical generation and neuron death. [Markesbery W.R, 1997, ref 25]

\section{Interrelation between amyloid-beta and mitochondria}

One of the most interesting events in $\mathrm{AD}$ is that mitochondrial oxidative stress occurs early in $\mathrm{AD}$ progression, before the onset of $\mathrm{A} \beta$ pathology. Mitochondria are the major source of ROS, and, in fact, mitochondrial dysfunction as well as hypometabolism has long been implicated in the onset of the familial and sporadic forms of AD. mtDNA defects have also been linked to an increased incidence of AD. Energy deficiency and mitochondrial dysfunction have been recognized as a prominent, early event in AD. Mitochondrial abnormalities have been found 
both in neurons and astrocytes, suggesting that both neurons and astrocytes might be damaged by free radicals in the AD brain. Superoxide radicals might be produced in mitochondrial electron transport chain complexes I and III and in components of the Krebs cycle, including $\alpha$ ketoglutarate dehydrogenas. In addition, superoxide radicals might be generated in the outer mitochondrial membrane. $\mathrm{H}_{2} \mathrm{O}_{2}$ and superoxide radicals, released from the mitochondrial matrix and from the inner and outer mitochondrial membranes, might be carried to the cytoplasm and, ultimately, might lead to the oxidation of cytoplasmic proteins. [28]

Both APP and A $\beta$ are present in mitochondrial membrane and interact with mitochondrial proteins, block mitochondrial import channels, impair mitochondrial transport, disrupt the electron transfer chain, increase ROS levels, and cause mitochondrial damage. [28]

APP and amyloid-beta may block mitochondrial translocation of nuclear-encoded proteins, such as components of the electron transport chain, impairing mitochondrial function. Intramitochondrial amyloid-beta is able to perturb mitochondrial function in several ways by directly influencing extracellular transport chain complex activities, impairing mitochondrial dynamics, or disturbing calcium storage, thus increasing apoptotic pathways. Moreover, amyloid-beta interacts with mitochondrial matrix components inducing an improper mitochondrial complex function leads to a decreased mitochondrial membrane potential of the organelle and impairing ATP formation. [28]

It is well documented that mtDNA changes are responsible for aging phenotypes.

It has been hypothesized that ongoing oxidative damage to mtDNA may be the underlying mechanism for cellular senescence. Since mtDNA repair mechanisms are limited and because mtDNA is situated in close proximity to the site of ROS production, mtDNA is more vulnerable to oxidative damage than nuclear DNA. With age, oxidation of mtDNA increases compared to nuclear DNA leading to an age dependent accumulation of mtDNA mutations. The effects of these mutations may lead to opening of the mitochondrial permeability transition pore and subsequent neuronal death. Increased ROS levels act at multiple levels to impair mitochondrial function: they induce mtDNA mutations that consequently negatively influence mitochondrial function, enhance amyloid-beta production by guiding APP cleavage pathway toward the amyloidogenesis, increase lipid peroxidation, activate mitophagy, leading to a reduced mitochondrial number, and augment tau hyperphosphorylation and NFT formation impairing organelle trafficking and neuronal function which leads to apoptosis. [28] In addition to its well-known role in glycolysis, GAPDH contributes to nuclear signaling in apoptosis. SNitrosylation of GAPDH terminates its enzymatic activity and allows binding of GAPDH to Siah1, an E3 ubiquitin ligase. Siah1 has a nuclear localization signal and carries GAPDH to the nucleus. GAPDH stabilizes Siah1 in the nucleus and allows degradation of nuclear proteins through ubiquitination. [27]

Protein-disulfide isomerase (PDI) is an endoplasmic reticulum (ER)-associated chaperone protein that prevents neurotoxicity caused by ER stress and protein misfolding and can also function as an NO receptor or donor, depending on the cellular context. PDI is nitrosylated both in AD and PD. Both PD and AD patient postmortem brains exhibit increased levels of nitrosylated PDI as compared with those of healthy controls. PDI nitrosylation prevents PDI-mediated ER stress reduction and allows protein misfolding. [27] 
Microglial activation is a key feature in Alzheimer's disease and is considered to contribute to progressive neuronal injury by release of neurotoxic products. Mononuclear phagocytes, such as microglial cells, are crucial components of the innate immune system. Cellular activation in response to pathogen-associated molecular patterns on microorganisms is mediated through interaction with innate immune receptors on the surfaces of mononuclear phagocytes, e.g., Toll-like receptors (TLRs) and the lipopolysaccharide receptor CD14. Lipopolysaccharide (LPS), a component of the cell wall of gram-negative bacteria, was first identified as the TLR ligand. The innate immune receptor Toll-like-receptor 4 (TLR4), localized on the surface of microglia, is a first-line host defense receptor against invading microorganisms. It has been shown that a spontaneous loss-of-function mutation in the Tlr4 gene strongly inhibits microglial and monocytic activation by aggregated Alzheimer amyloid peptide resulting in a significantly lower release of the inflammatory products IL-6, TNF $\alpha$ and nitric oxide. Treatment of primary murine neuronal cells with supernatant of amyloid peptide-stimulated microglia demonstrates that Tlr4 contributes to amyloid peptide-induced microglial neurotoxicity. The reason how TLR4 becomes activated in the pathophysiology of AD is unclear. These findings further support a role of TLR4 in neuroinflammation in AD. Microglial activation in AD pathophysiology is discussed as a two-edged sword. [29]

It has been demonstarted that $\mathrm{A} \beta 1-40$ administration induced an increase in TNF- $\alpha$ expression and oxidative alterations in prefrontal cortex and hippocampus. Likewise, A $\beta 1-40$ led to activation of both JNK (c-Jun-NH2-terminal kinase)/c-Jun and nuclear factor- $\kappa \mathrm{B}$, resulting in iNOS upregulation in both brain structures. [29] It has been shown that the anti-TNF- $\alpha$ antibody reduced all of the molecular and biochemical alterations promoted by A $\beta 1-40$. These results provide new insights in Mouse models of AD, revealing TNF- $\alpha$ and iNOS as central mediators of $\mathrm{A} \beta$ action. These pathways might be targeted for AD drug development. [29]

Tumor necrosis factor- $\alpha$ (TNF- $\alpha$ ) is a cytokine thought to play a central role in the selfpropagation of neuroinflammation. TNF- $\alpha$ regulates many cellular processes, including inflammation, differentiation, and cell death through activation of TNF receptor 1 (TNFR1) or TNFR2. The transduction pathways activated by TNF- $\alpha$ include mitogen-activated protein kinases (MAPKs) and IкB kinase, which control gene expression through transcriptional factors such as activator protein-1 (AP-1) and nuclear factor- $\kappa \mathrm{B}(\mathrm{NF}-\mathrm{kB})$. Regarding the CNS, microglia and astrocytes are believed to be the primary sources of TNF- $\alpha$. Evidence indicates the presence of increased levels of TNF- $\alpha$ in the brain and plasma of AD patients and an upregulation of TNFR1 have been detected in the AD brain. A $\beta$ has been shown to interact in a synergistic manner with cytokines to induce neuronal damage via reactive oxygen species (ROS)- and NO-dependent pathways. [30] Numerous animal models have been used to evaluate the role of inflammation in the course of $\mathrm{AD}$. An experimental model that mimics the progression of $\mathrm{AD}$ was developed using an intracerebroventricular injection of $\mathrm{A} \beta$ in mice. The cross talk between TNF- $\alpha$ and iNOS is probably mediated by activation of two major intracellular pathways: c-Jun-NH2-terminal kinase (JNK)/c-Jun and NF- $\kappa B$. According to the results, they suggested that TNF- $\alpha$ and iNOS as important mediators of $A \beta$-induced cognitive impairment. TNF- $\alpha$ signaling effectors include JNK and c-Jun that might also contribute to iNOS promoter activity. [29] 


\section{A $\beta$ and age}

The glycation hypothesis of aging suggests that modification of proteins by glucose (the "Maillard reaction") leads to the development of "advanced glycation end-products"

(AGEs). [31] In the human brain, AGEs accumulate in neuronal perikarya of the hippocampus and parahippocampus, as well as in reactive astroglia in brains after the third decade of age. In $\mathrm{AD}$, this effect is twofold: AGEs accumulate extracellularly on $\beta$-amyloid plaques and intracellulary in neurons and astrocytes. The binding of AGEs to its receptor, RAGE (receptor for advanced glycation endproducts), activates NADPH-oxidase, a central participant in the production of superoxide radicals. Superoxide and its conversion product, hydrogen peroxide, were shown to activate redoxsensitive transcription factors such as NF- $\kappa B$ and activator protein 1 (AP-1) resulting in the upregulation of cytokines such as interleukin (IL)-1, IL-6, tumor necrosis factor (TNF- $\alpha$ ) and inducible nitric oxide synthase (iNOS). Interestingly, RAGE is also activated by $\mathrm{A} \beta$, the major pro-inflammatory peptide present in amyloid plaques in $\mathrm{AD}$ and HMGB1/amphoterin, a novel pro-inflammatory ligand released from dying cells. TNF- $\alpha$ and $\mathrm{NO}$ were chosen as relevant biomolecules due to their role in augmenting inflammation and/or inducing cell death. TNF- $\alpha$ (cachexin or cachectin) is a cytokine involved in systemic inflammation that upregulates other NF- $\kappa \mathrm{B}$-regulated cytokines and is also a member of a group of cytokines that stimulate the acute phase reaction. TNF- $\alpha$ is also able to induce apoptotic cell death. [31]

Although the exact target of the polyphenols along the pro-inflammatory signal cascades is not known in detail, it is most likely suggested that they interfere with the NF- $\kappa B$ pathway somewhere upstream of kinases phosphorylating the inhibitor of $\kappa \mathrm{B}, \mathrm{I} \kappa \mathrm{B}$. There are two signaling pathways leading to the activation of NF- $\kappa B$ known as the canonical pathway (or classical) and the noncanonical pathway (or alternative pathway). [31] The common regulatory step in both of these cascades is activation of an I $\kappa \mathrm{B}$ kinase $(\mathrm{I} \kappa \mathrm{K})$ complex consisting of catalytic kinase subunits ( $\mathrm{I} \kappa \mathrm{K} a$ and/or I $\kappa \mathrm{Kb}$ ) and the regulatory nonenzymatic scaffold protein NEMO. Activation of NF- $\kappa \mathrm{B}$ dimers is due to I $\kappa \mathrm{K}$-mediated phosphorylation-induced proteasomal degradation of the I $\kappa \mathrm{B}$ inhibitor enabling the active NF- $\kappa \mathrm{B}$ transcription factor subunits to translocate to the nucleus and induce target gene expression. In the canonical (or classical) activation pathway, the complex consisting of I $\kappa \mathrm{K}-\mathrm{b}$ and I $\kappa \mathrm{K}-\mathrm{g} / \mathrm{NEMO}$ phosphorylates two

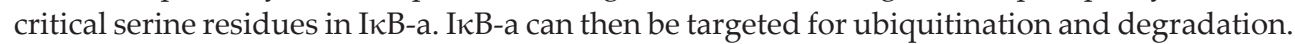
Some non-canonical pathways of I $\kappa \mathrm{K}$-independent activation of NF- $\kappa$ B stipulate the selective activation of NF- $\kappa$ B subunits. [31]

\section{Deregulation of intracellular signallings due to $A \beta$}

The studies suggest that various intracellular signaling pathways are deregulated in AD brains or during A $\beta$-induced neuronal apoptosis. For example, activation of stress-related kinases cJun N-terminal kinase (JNK) and p38 is associated with neuronal death in AD Mouse model. [32] Glycogen synthase kinase 3 (GSK-3) has also been implicated in A $\beta$-induced neurotoxicity. 
Deregulation of distinct signaling pathways leads to aberrant phosphorylation of cellular proteins and has a profound effect on the progression of AD. [32]

They reported the identification of signal transducer and activator of transcription 3 (STAT3) as a potential key player in AD pathophysiology. STAT3 is a transcription factor that is typically associated with cytokine signaling during neuronal differentiation, inflammation, and malignancies. Interestingly, they found that tyrosine phosphorylation of STAT3, which is required for the activation of this transcription factor, is markedly elevated in neurons treated with A $\beta$ in vitro or in vivo as well as in the brains of APP/PS1 transgenic mice. Inhibition of STAT3 activation or reduced STAT3 expression significantly attenuates A $\beta$-induced neuronal cell death. Moreover, activation of a tyrosine kinase Tyk2 is required for the A $\beta$-induced tyrosine phosphorylation of STAT3 and neuronal cell death. Notably, elevation of STAT3 tyrosine phosphorylation is evident in postmortem samples of AD brains. These observations collectively raise an intriguing possibility that STAT3 signaling is involved in neuronal apoptosis observed in AD patients. [32]

\section{Interactions between $\mathrm{A} \beta$ and microglia}

Activated microglia secrete various humoral substances (cytokines, free radicals) which influence neurons and glia. The inhibition of C3 conversion resulted in the lack of $A \beta$ opsonisation and prevented microglia from $A \beta$ clearance and, as a result, doubled the amyloid burden in murine brains. [33] The following may be responsible for the inconsistency in the results of experiments on microglia behaviour in contact with $A \beta$ :

- immunomodulation of astroglia,

- immunomodulation of neurons,

- senescence of microglia.

Microglia activation is followed by astrocyte activation. Astrocytes phagocyte and degrade $\mathrm{A} \beta$. In vivo experiments suggest that the coexistence of microglia and astrocytes diminishes the ability of microglia to digest and degrade plaques and $\mathrm{A} \beta$. Therefore, activated astrocytes may exert a regulative effect (negative feedback) on the phagocytic activity of microglia. Another factor influencing microglia activation is the neuronal expression of cyclooxygenase-2 (COX-2). COX-2 participates in prostaglandin production and its expression is usually elevated in places of inflammation. [33]

In AD, initially COX-2 expression is evident in pyramidal neurons particularly involved in AD. COX-2 expression rises at the onset of the disease and then declines in the advanced stages of AD. Of note, the expression of COX-2 correlated positively with the level of prostaglandin E2 (PGE2) in cerebrospinal fluid (CSF). CSF PGE2 levels are clearly higher in people with mild dementia and decrease in the late stages of AD. [33]

Together with astrocytes, microglia have the ability to bind and take up $A \beta$ in vitro and in vivo, being important players for the deposition and removal of $A \beta$, although there are also 
reports suggesting that $A \beta$ formation occurs independently of microglial cells. [34] Microglial cells express several pattern-recognition receptors, which allow them to remove potentially toxic molecules such as $A \beta$. These receptors include scavenger receptors (SR) class A (SR-A), class B type I, low-density lipoprotein receptor-related protein (LRP), cluster of differentiation 36 (CD36), receptor for end glycation product, mannose receptor, and SR-MARCO. [34]

In fact, microglial cells secrete multiple cytokines such as interleukin-1b (IL1b), tumor necrosis factor (TNF- $\alpha$ ), interleukin 10 (IL10), and transforming growth factor-b (TGF $\beta$ ) as well as shortlived cytotoxic factors such as superoxide $\left(\mathrm{O}_{2}^{-2}\right)$ and $\mathrm{NO}$, which contribute to neurotoxicity. [34] It has been suggested that TGF $\beta 1$ produced by astrocytes and hippocampal cells prevents induction of reactive oxygen species (ROS) and $\mathrm{NO}^{-}$production by inflammatory mediators (lipopolysaccharide [LPS] 1 interferon- $\gamma$ [IFN $\gamma]$ ) and neurotoxicity in vitro and that the modulation of microglia is at least partially mediated by the activation of the ERK pathway. TGF $\beta$ superfamily signaling plays important roles in a diverse set of cellular responses, including cell proliferation, differentiation, extracellular matrix remodeling, and embryonic development. TGF $\beta 1$ signaling is mediated by cell surface type I and type II receptors, which phosphorylates the two R-Smad proteins Smad2 and Smad3 downstream. Phospho-Smad2/3 forms a complex with the common mediator Smad4 that binds to a Smad-binding element (SBE) in the nucleus with a large number of transcription coregulators to activate gene promoter. They showed that the TGF $\beta 1$ Smad3 pathway is involved in modulation of microglial cell activity through its effects on expression of SRs, NO- secretion, and phagocytosis. Microglial cell activation can be initiated by various signal transduction pathways, including nuclear factor-jB, JAK/STAT, and p38 pathways, which are activated by several inflammatory mediators and $\mathrm{A} \beta$. [34]

\section{9. $A \beta$ and apoptosis}

Apoptosis, or programmed cell death, is a highly regulated process involved in embryonic development, developmental tissue remodeling and normal cell turnover. [35] However, when dysregulation occurs in apoptotic pathways, excessive or insufficient cell death can lead to diseases such as cancers, autoimmune syndromes and/or neurodegenerative diseases. Caspases are a family of intracellular cysteine-aspartic proteases that are not only essential for triggering programmed cell death, but have also been shown to play key roles in non-apoptotic pathways, such as differentiation and proliferation of diverse cell types, axon guidance and synaptic activity and plasticity. Caspases are divided into long prodomain caspases (caspases-2, -8, -9 and -10), which are initiators of apoptosis, and short prodomain caspases (caspases-3, -6, -7 and -14), which are generally termed the effectors of apoptosis. However, some caspases, including caspase-3 (Casp3) and caspase-6 (Casp6), appear to function as both initiators and effectors. Aberrant activation of caspases has been implicated in several neurodegenerative diseases, such as $\mathrm{AD}, \mathrm{HD}$, various ataxias and amyotrophic lateral sclerosis [35].

Apoptosis is a cell death program that is central to cellular and tissue homeostasis, and is involved in many physiological and pathological processes. [36] Apoptosis is characterized 
morphologically by a series of events that include cytoplasmic shrinkage, chromatin condensation, nuclear and cellular fragmentation, and the formation of apoptotic bodies. Although caspases are the main players involved in apoptosis, there are other molecules involved in the progression of the apoptotic cascade that are relevant to AD. The neuronal death in AD may result directly and/or indirectly from the triggering insults caused by $\mathrm{A} \beta$ toxicity, glutamate excitotoxicity, long-lasting oxidative stress, DNA damage, and elevation of intracellular calcium levels. Thus, the mode of cell death in AD remains a matter of controversy, and it is possible that both apoptotic and non-apoptotic cell death coexist in the brains of affected patients. [36]

Previously, it was generally considered that apoptotic neuronal death in chronic neurodegenerative disease, e.g., AD, Parkinson's disease, etc., is associated with classical caspase mediated cell death. However, in part, it was suggested that the caspase-independent pathway might also participate in the pathogenesis of the disease. Cross talk is extensive between different cell death pathways, which include multiple types of caspase-dependent and caspase-independent programmed cell death. [36]

Cells undergo apoptosis through two major pathways, the extrinsic pathway (death receptor pathway) and the intrinsic pathway (the mitochondrial pathway). These two pathways can be linked by caspase-8-activated truncated Bid formation. Very recently, death receptor 6 (DR6) was shown to be involved in the neurodegeneration observed in Alzheimer disease. DR6, also known as TNFRSF21, is a relatively new member of the death receptor family, and it was found that DR6 induces apoptosis when it is overexpressed. However, how the death signal mediated by DR6 is transduced intracellularly is not known. To this end, in a study they have examined the roles of caspases, apoptogenic mitochondrial factor cytochrome c, and the Bcl-2 family proteins in DR6-induced apoptosis. In their study results demonstrated that Bax translocation is absolutely required for DR6-induced apoptosis. On the other hand, they found inhibition of caspase-8 and knockdown of Bid have no effect on DR6-induced apoptosis. Their results strongly suggest that DR6-induced apoptosis occurs through a new pathway that is different from the type I and type II pathways through interacting with Bax. [37]

Different studies reported both necrotic and apoptotic mechanisms for A $\beta$-mediated neurotoxicity. In particular, oxidative-mediated DNA damage, with a pattern indicative of apoptosis, was found in AD brain, which is consistent with several lines of experimental evidence linking oxidative stress and neuronal apoptosis. [38] Apoptosis is induced by micromolar concentrations of $\mathrm{A} \beta$ in cultured CNS neurons, however, physiological nanomolar concentrations of $A \beta 1-40$ and $A \beta 1-42$ are insufficient to initiate significant apoptosis in cultures of human fetal neurons. In fact, both $A \beta$ peptides downregulate "bcl-2", a key anti- apoptotic protein, while only A $\beta 1-42$ upregulates "bax", a protein known to promote apoptotic cell death. Interestingly, $A \beta$ treated neurons exposed to different levels of oxidative stress, unable to increase apoptosis in control neurons, show 10-20 times more apoptotic-mediated DNAdamage, suggesting that $A \beta$ renders the neurons vulnerable to age-dependent oxidative stress and neurodegeneration. Other links between oxidative stress and apoptotic neuronal cell death in AD have been described. Apoptosis induced by 4-HNE is prevented in cells that overexpress "bcl-2" or by incubation with glutathione, which binds 4-HNE. Also, PC12 cells expressing a 
mutated presenilin-1 gene, which accounts for the majority of cases of inherited early onset forms of $\mathrm{AD}$ are more susceptible to apoptosis induced by micromolar concentrations of $\mathrm{A} \beta$ and to oxidative stress induced by nerve growth factor with- drawal. [38]

Microglial activation can lead to microglial apoptosis, which may serve to remove highly reactive and possibly neurotoxic microglia. [39] However the loss of microglia may have consequences for future recovery, protection and repair. P53, a nuclear phosphoprotein transcription factor, is critical for activating the expression of genes involved in cell-cycle arrest and stress-induced apoptosis. In neurodegenerative diseases the expression of p53 is significantly increased in glial cells, and microglial numbers fall. P53 is a transcription factor, the activation of which promotes cellular apoptosis through the normal cell cycle. [39]

P53 also activates the expression of genes involved in stress-induced apoptosis and the apoptotic pathways implicated in neurodegenerative diseases which may arise from inappropriate p53 activation. Changes in p53 expression occur in glial cells in neurological conditions; p53 expression increases predominantly in glial cells in Alzheimer's disease. The sustained reactivity of microglia is implicated in the pathology of many neurodegenerative diseases and microglia may secrete substances which compromise neighbouring cells. Increased microglial reactivity can lead to microglial apoptosis. This apoptosis results from microglial stress and is triggered by nitric oxide-dependent mitochondrial depolarization and caspase activation. Whilst microglial apoptosis may serve to limit the number of reactive microglia in the brain, the processes of microglial stress and apoptosis can in themselves result in the release of proapoptotic species such as soluble Fas ligand which can trigger bystander cell apoptotic cascades. Microglial numbers significantly decrease in aged human brain and in Alzheimer's brain and such loss may restrict the ability of the brain to recover from injury. Apoptotic changes in microglia precede changes in neurons or other glia in models of neurodegenerative disease. As microglia secrete neurotrophins important for neuronal survival and regeneration, the apoptosis of microglia may impact on brain health. They examined the role p53 plays in mediating microglial apoptosis following microglial activation with the Alzheimer peptides amyloid beta and chromogranin A, as well as with the activator lipopolysaccharide. [39]

Taken together, these results suggest that mitochondrial energy metabolism might be impaired by $\mathrm{A} \beta$ through down-regulation of mitochondrial proteins and activity. [40] In addition, the interaction between $\mathrm{NO}$ and cytochrome $c$ oxidase controls mitochondrial production of hydrogen peroxide, which has been shown to be implicated in cellular redox signaling. In a study they used synthetic A $\beta 1-42$ to impair the function of complexes I and IV in CP epithelial cells and to investigate whether ROS generation was also altered. [40] Their results support the hypothesis that A $\beta 1-42$ interferes with oxidative phosphorylation, which results in oxidative stress, and demonstrate that ROS generation is secondary to mitochondrial damage. The results of the study also show that A $\beta 1-42$-treated CP epithelial cells have an increased expression of caspases 3 and 9. [40] This result is consistent with a recent study, where APP transfected cells showed a significant increase in the expression and activity of caspases 3 and 9. The data suggest that the excessive generation of ROS would be responsible for initiating the apoptotic cell death process by up-regulating caspase signaling, as previously demonstrated. In view of the results from their in vitro and in vivo studies, they proposed that $\mathrm{A} \beta-$ 
induced mitochondrial dysfunction provides an important source of ROS, which could ultimately initiate a programmed cell death pathway. In CP from APP/PS1 mice and AD patients, this pathway involves high levels of MMP-9, increased caspases expression and cell loss. [40]

Previous studies found that expression levels of Bcl-2 family proteins, such as Bax, Bak, Bad, $\mathrm{Bcl}-2$, Bim, Bcl-w and Bcl-x are altered in the vulnerable neurons in AD. [41] They demonstrated that oligomeric $\mathrm{A} \beta$ altered the expression levels of $\mathrm{Bcl}-2$, Bim and $\mathrm{Bax}$, and that the genetic or pharmacological ablation of Bax activity suppresses oligomeric $A \beta$-mediated neurotoxicity in both ex vivo and in vivo. These results clearly indicate that Bax has an essential role in the induction of neuronal cell death caused by oligomeric A $\beta$. [41] In healthy cells, Bax is located in the cytosol or loosely associated to mitochondria and endoplasmic reticulum. Bax translocation to the mitochondria, which occurs in cells with apoptotic stresses, is thought to lead to mitochondrial dysfunction and release of cytochrome $\mathrm{c}$ and subsequent apoptosis. Before its translocation to mitochondria, Bax changes its conformation that exposes the N-terminal residues. This conformational change is believed to be necessary for membrane insertion of Bax at mitochondria and multimerization of Bax. They demonstrated that oligomeric A $\beta$ induced the N-terminal exposure of Bax in neurons and that the inhibition of this event by BIP rescues neurons from oligomeric $A \beta$ 's neurotoxicity, suggesting the activation of Bax by its conformational change is a key element of oligomeric $A \beta$-induced neurotoxicity. Although the molecular mechanism(s) of Bax activation has not been clearly defined, multiple pro-apoptotic proteins (e.g.,Bim) and anti-apoptotic proteins (e.g., Bcl-2) are known to regulate the activation of Bax thorough heterodimerization. These data strongly suggest that Bim might be an upstream regulator of Bax activation in oligomeric $A \beta$-induced neurotoxicity and the pathological role of Bim and Bax in neuronal cell loss in AD. [41]

As the activation of JNK by A $\beta$ has been shown and the active form of JNK has been reported to be increased in vulnerable neurons in $A D$, it is plausible that the activation of JNK and subsequent activation of Bim/Bax-mediated apoptosis pathway might be the mechanism causing neuronal cell death in oligomeric $\mathrm{A} \beta$ treated neurons and AD. [41]

As the chronic administration of resveratrol also protected animals from $A \beta$-induced neuronal loss, it is reasonably speculated that the improvement in spatial memory by resveratrol might be ascribed to its effectiveness in reducing the levels of oxidative stress and the A $\beta$-induced neuronal loss. [42] The activation of iNOS gene by $A \beta$ is controlled by the transcription factor $\mathrm{NF}-\kappa \mathrm{B}$, which is known to regulate iNOS transcription by binding to the regulatory region of iNOS gene. In addition, $A \beta$ treatment was reported to result in the activation of NF- $\kappa B$ in various brain regions and cell types, and resveratrol was shown to markedly attenuate the $A \beta-$ induced $N F \kappa B$ nuclear translocation. Based on these, it is suggested that the regulation pathway triggered by resveratrol is to decrease the $A \beta$ accumulation, which in turn suppresses $\mathrm{A} \beta$-induced NF- $\mathrm{B}$ translocation, or activation, and leads to the downregulation of iNOS. [42]

Considering the strong correlation between $\mathrm{A} \beta$-induced oxidative stress and cytotoxicity, ROS produced in mitochondria may leak to the cytoplasm, leading to oxidative stress and the initiation of apoptosis via the activation of apoptosis signaling. [43] It is well established that the ratio of $\mathrm{Bcl}-2 / \mathrm{Bax}$ is crucial in the apoptosis of the mitochondrial pathway. Bcl-2 is a potent 
cell death suppressor, and its overexpression prevents cell death. However, Bax is a deathpromoting factor, and its translocation to the mitochondrial membrane may lead to the loss of mitochondrial membrane potential and an increase in mitochondrial permeability. Increased mitochondrial permeability results in the egress of cytochrome $c$ from the mitochondria and the subsequent activation of procaspase- 3 to caspase- 3 , which eventually leads to apoptosis. $\mathrm{A} \beta$ treatment significantly decreased the Bcl-2/Bax protein expression ratio and increased caspase-3 activity, in agreement with previous reports. [43]

Classic programmed cell death is more frequently associated with a death program that requires gene transcription and protein synthesis, whereas apoptosis is generally independent of protein synthesis and represents a posttranslational response of host cells. Apoptosis is characterized by a requirement for specific proteolysis driven by caspases, although it is also reported that caspases (caspase 9 in particular) participate in nonapoptotic cell death. [35]

The name caspase is a contraction of cysteinedependent aspartate-specific protease; their enzymatic properties are governed by a dominant specificity for substrates containing Asp and by the use of a Cys side chain for catalyzing peptide bond cleavage. Mammals contain two biologically distinct caspase subfamilies: One of these participates in the processing of proinflammatory cytokines, and the other is required to elicit and execute the apoptotic response during programmed cell death. [35]

The intrinsic pathway responds primarily to cellular stress (ionizing radiation, cytotoxic drugs, etc.) as well as some neurodevelopmental cues, with the mitochondrion acting as an important integrator. Activation of the apical protease caspase 9 occurs when it is driven into a catalytic conformation by its cofactor Apaf-1, which itself requires prior binding to cytochrome $\mathrm{c}$. The extrinsic apoptosis pathway is triggered through the extracellular ligation of death receptors by their cognate ligands, resulting in receptor clustering, adapter recruitment, and activation of the apical protease caspase 8. [35]

The in vivo models offer the advantage of allowing neuronal death to proceed in a more physiologic setting, where the different components of the nervous system are intact. The in vivo models, however, do not adequately allow determination of the specific caspases necessary to execute neuronal death in each disease. [35] Elucidation of the specific caspase pathways is best approached in the cell culture models, with which biochemical studies can be more easily performed. Cell culture models include both primary neuronal cultures and cell lines. Both are valid models, as long as their limitations are appreciated. Cell lines offer the advantage of providing large amounts of homogenous material in which expression levels of the different components of the death pathways can be easily manipulated. Results from cell lines must normally be validated in vivo in the cell that they are modeling. After establishment of the potential caspase pathways in the cell culture models, the in vivo and human samples can be analyzed. [35] Caspases are a family of cysteine proteases that cleave their substrates after aspartic residues. They are usually synthesized as inactive zymogens that are proteolytically cleaved into subunits at the onset of apoptosis and function as active caspases after reconstitution to molecular heterodimers. [45] Caspases are composed of three domains including an N-terminal prodomain, a large subunit, and a small subunit. The apoptoticassociated caspases include initiator caspases, such as caspases 2, 8, 9, and 10, that activate 
downstream executioner caspases, resulting in an amplification of cascade activity. The initiator caspases consist of long N-terminal prodomains that contain caspase recruitment domains (CARDs) in caspase 2 and caspase 9, or death effector domains (DEDs) in caspase 8 and caspase 10. Another set of caspases, termed the executioner caspases, consists of caspases 3,6 , and 7 that function to directly cleave crucial cellular protein substrates that result in cell destruction. [45] The executioner caspases contain short prodomains or have no prodomains. Apoptotic injury during Alzheimer's disease may require caspase-mediated pathways. A strong body of evidence supports the premise that caspase activation is involved in the pathological process of Alzheimer's disease. The elevation of caspase genes including caspases $1,2,3,5,6,7,8$, and 9 has been observed in human postmortem brains from Alzheimer's disease patients. In the brains of Alzheimer's patients, single neurons with DNA fragmentation have been shown to contain cytoplasmic immunoreactivity for active caspase 3, implying that apoptotic injury results during Alzheimer's disease. In addition, activation of caspase 3 was found to occur in the parahippocampal gyrus in brains from patients with mild forms of Alzheimer's disease. Caspase 3 immunoreactivity was also co-localized with paired helical filaments in neurons, suggesting that caspase 3 activation may contribute to the formation of neurofibrillary tangles. [45]

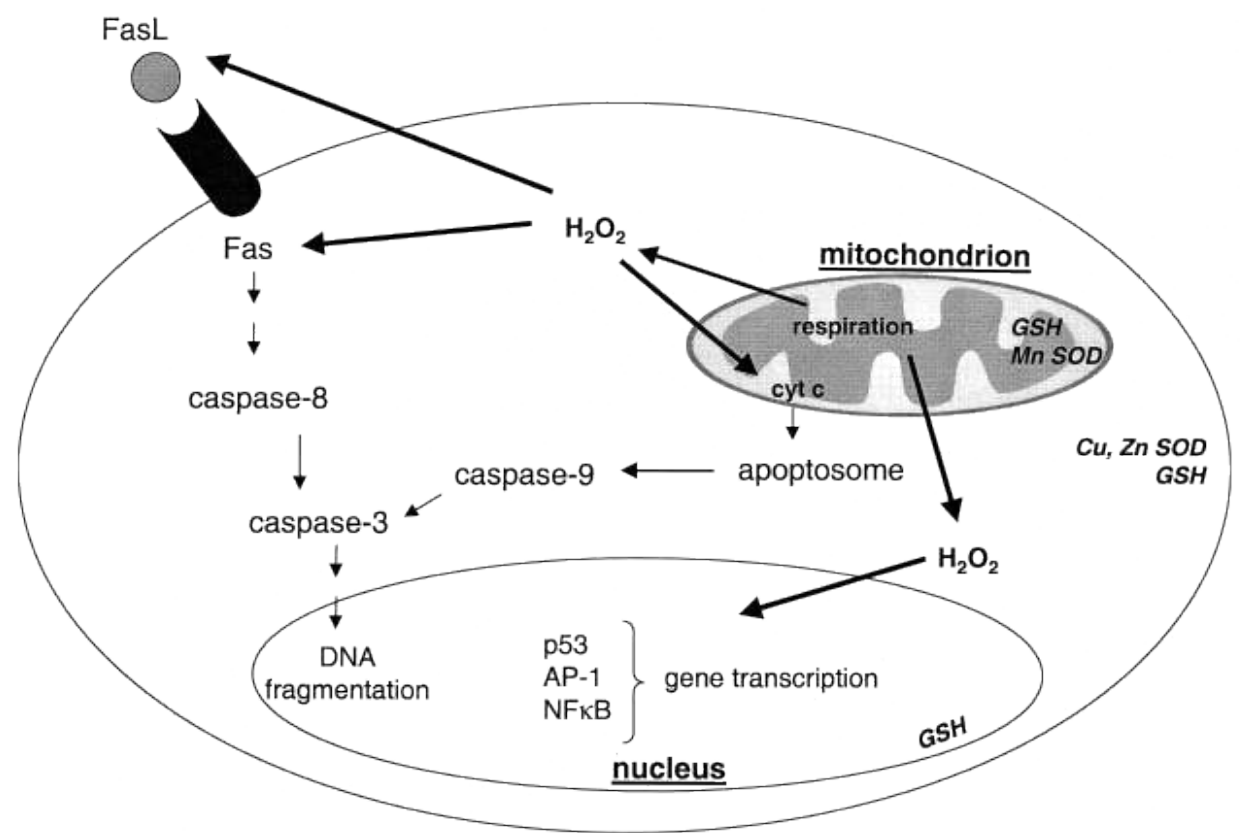

Figure 3. Intracellular sources of ROS and their interaction with the apoptotic pathway. [Chandra J. 2000, ref 26]

As $\mathrm{A} \beta$ peptides are used to mimic $\mathrm{AD}$ in animal models, and because sporadic $\mathrm{AD}$ is known to arise mostly in older ages, we designed a study to investigate the acute effects of $A \beta 1-42$ 
injection in aged rats suggesting the onset of sporadic $\mathrm{AD}$. In the light of the current literature, we investigated not only the effect of $A \beta 1-42$ injection on aged rat brain, but also age related changes in lipid peroxidation, nNOS and iNOS expression and caspase 3 in which involves apoptotic process as an apoptosis marker. In this study, a prominent increase of caspase 3 activity in hippocampus has been shown in A $\beta$ 1-42 injected aged rats. [24] This result suggests that apoptotic mechanism triggers neuronal death in hippocampus, and results a significant decrement nNOS expression consequently in A $\beta$ 1-42 injected aged rats. Another explanation of these results is that because of possible neuronal death by the way of caspase- 3 , there may not be enough neurons remained to cause lipid peroxidation. This suggests that acute effect of $A \beta$ 1-42 result with increase in caspase 3 activity which is seen before the oxidative stressdependent neurotoxic effects. In this study A $\beta$ 1-42 lead to a counter effect on nNOS expression in young adults and aged rats. The results of this study suggest that the aged rat brain does not successfully offset the oxidative stress. [24] We suggest that the relationship between aging, NOS-mediated ROS-dependent toxicity of beta amyloid toxicity and apoptotic events should be bring out into the open this vicious cycle.

\section{Abbreviations}

ROS: Reactive oxygen species

AD: Alzheimer's disease

A $\beta$ : Beta amyloid peptide

NO: Nitric oxide

NOS: Nitric oxide synthase

CA: Cornu ammonis

$\mathrm{H}_{2} \mathrm{O}_{2}$ : Hydrogen peroxide

ONOO: Peroxynitrite

APP: Amyloid precursor protein

RNS: Reactive nitrogen species

GADPH: Glucose 6-phosphate dehydrogenase

MAPK: Mitogen activated protein kinase

AP-1: Activated protein

NFкB: Nuclear factor kapa B

CNS: Central nervous system

AGE: Advanced glycation end product

I $\kappa$ : Inhibitor of $\kappa \mathrm{B}$ 
I $\kappa$ BK: Inhibitor of $\kappa B$ kinase

JNK: c-Jun N-terminal kinase

GSK-3: Glycogen synthase kinase 3

STAT3: Signal transducer and activator of transcription 3

Tyk2: tyrosine kinase 2

SR: Scavenger receptor

LRP: Low-density lipoprotein receptor related protein

TGF: Transforming growth factor

SBE: Smad binding element

HD: Huntington disease

PD: Parkinson disease

DR6: Death receptor 6

SOD: Superoxide dismutase

GSH: Glutathione

\section{Author details}

Ferihan Cetin ${ }^{*}$

Address all correspondence to: ferihan@yahoo.com

Izmir University Faculty of Medicine, Department of Physiology, Medical Park Hospital, Izmir, Turkey

\section{References}

[1] Beckman, K. B, \& Ames, B. N. The free radical theory of aging matures. Physiol Rev. (1998). , 78, 547-581.

[2] Sastre, J, \& Pallardo, F. V. Mitochondrial oxidative stress plays a key role in aging and apoptosis. IUBMB life (2000). , 49427-435.

[3] Fiala, M, Cribbs, D. H, Rosenthal, M, \& Bernard, G. Phagocytosis of amyloid-beta and inflammation: two faces of innate immunity in Alzheimer's disease. J Alzheimers Dis. (2007). , 11(4), 457-63. 
[4] Yamada, K, \& Nabeshima, T. Animal models of Alzheimer's disease and evaluation of anti-dementia drugs. Pharmacol. Therap. (2000). , 88-93.

[5] Camandola, S, Poli, G, \& Mattson, M. P. Lipid Peroxidation Product 4-hydroxynonenal increases AP-1 binding activity through caspase activation in neurons. J Neurochem. (2000). , 74(1), 159-68.

[6] Stepanichev, M. Y, Onufriev, M. V, Yakovlev, A. A, Khrenov, A. I, \& Peregud, D. I. Amyloid- $\beta$ (25-35) increases activity of neuronal NO-synthase in rat brain. Neurochemistry International (2008). , 52-1114.

[7] Law, A, Gauthier, S, \& Quiron, R. Say NO to Alzheimer's disease: putative links between nitric oxide and dementia of the Alzheimer's type. Brain Res. Rev. (2001). , 35-73.

[8] Xie, X, Wang, H-T, Li, H-L, Gao, X-H, Ding, J, \& Zhao, H-H. Ginsenoside Rb1 protects PC12 cells against $\beta$-amyloid-induced cell injury Mol Med Reports (2010). , 3, 635-639.

[9] Christen, Y. Oxidative stress and Alzheimer disease. Am J Clin Nutr (2000).

[10] Lovell, M. A, Ehmann, W. D, Butler, S. M, \& Markesberry, W. R. Elevated thiobarbituric acid-reactive substances and antioxidant enzyme activity in the brain in Alzheimer's disease. Neurology (1995). , 45-1594.

[11] Machiavelli, L. I, Poliandri, A. H, Quinteros, F. A, Cabilla, J. P, \& Duvilanski, B. H. Reactive oxygen species are key mediators of the nitric oxide apoptotic pathway in anterior pituitary cells. Nitric Oxide Mar (2007). , 16(2), 237-46.

[12] Vina, J, Lloret, A, Vallés, S. L, Borrás, C, Badía, M. C, Pallardó, F. V, \& Sastre, J. Mitochondrial oxidant signalling in Alzheimer's disease. J Alzheimers Dis. (2007). , $11(2), 175-81$.

[13] Feng, Z, Qin, C, Chang, Y, \& Zhang, J. Early melatonin supplementation alleviates oxidative stress in a transgenicmouse model of Alzheimer's disease. Free Rad Biol \& Med (2006). , 40-101.

[14] Thorns, V, Lawrence, H. L, \& Masliah, E. nNOS Expressing Neurons in the Entorhinal Cortex and Hippocampus Are Affected in Patients with Alzheimer's Disease. Exper Neurol (1998). , 150-14.

[15] Norris, P. J, Faull, R. L, \& Emson, P. C. Neuronal nitric oxide synthase (nNOS) mRNA expression and NADPH-diaphorase staining in the frontal cortex, visual cortex and hippocampus of control and Alzheimer's disease brains. Brain Res Mol Brain Res. (1996).

[16] Koliatsos, V. E, Kecojevic, A, Troncoso, J. C, Gastard, M. C, Bennett, D. A, \& Schneider, J. A. Early involvement of small inhibitory cortical interneurons in Alzheimer's disease. Acta Neuropathol (2006). , 112-147.

[17] Butterfield, D. A, \& Lauderback, C. M. Lipid peroxidation and protein oxidation in Alzheimer's Disease brain: potential causes and consequences involving Amyloid $\beta$ - 
Peptide-associated free radical oxidative stress. Free Rad Biol Med. (2002). , 32(11), 1050-1060.

[18] Jhoo, J. H, Kim, H-C, Nabeshima, T, Yamada, K, Shin, E-J, Jhoo, W-K, \& Kim, W. Amyloid (1-42)-induced learning and memory deficits in mice: involvement of oxidative burdens in the hippocampus and cerebral cortex. Behav Brain Res (2004). , 155, 185-196.

[19] Siles, E, Martinez-lara, E, Canuelo, A, Sánchez, M, Hernández, R, \& López-ramos, J. C. $\mathrm{M}$, Age related changes of the nitric oxide system in the brain. Brain Res. November (2002). , 956(2), 385-92.

[20] Koppal, T, Drake, J, Yatin, S, Jordan, B, Varadarajan, S, Bettenhausen, L, \& Butterfield, D. A. Peroxynitrite-induced alterations in synaptosomal membrane proteins: insight into oxidative stress in Alzheimer's Disease. J. Neurochem. (1999). , 72, 310-317.

[21] Malinski, T. Nitric oxide and nitro-oxidative stress in Alzheimer's Disease. J.Alz. Disease. May (2007). , 11(2), 207-18.

[22] ] Maccioni, R. B, Muñoz, J. P, \& Barbeito, L. The Molecular Bases of Alzheimer's Disease and Other Neurodegenerative Disorders. Archives of Medical Research (2001). , 32, 367-381.

[23] Weldon, D. T, Rogers, S. D, Ghilardi, J. R, Finke, M. P, Cleary, J. P, Hare, O, Esler, E, Maggio, W. P, Mantyh, J. E, \& Fibrillar, P. W. Amyloid Induces Microglial Phagocytosis, Expression of Inducible Nitric Oxide Synthase, and Loss of a Select Population of Neurons in the Rat CNS In Vivo The Journal of Neuroscience, March 15, (1998). , 18(6), 2161-2173.

[24] Cetin, F, Yazihan, N, Dincer, S, \& Akbulut, K. G. The The Effect of Intracerebroventricular Injection of Beta Amyloid Peptide (1-42) on Caspase 3 Activity, Lipid Peroxidation and NOS expression in Young Adult and Aged Rat Brain. Turkish Neurosurgery (2012). date accepted May 17.DOİ. :10.5137/JTN.5855-12.1, 1019-5149.

[25] Markesbery William ROxidative stress hypothesis in Alzheimer's Disease. Free Radical Biology \& Medicine, (1997). , 23(1), 134-147.

[26] Chandra, J, Samali, A, \& Orrenius, S. Triggering and modulation of apoptosis by oxidative stres. Free Radical Biology \& Medicine, (2000). Nos. 3/4, , 29, 323-333.

[27] ] Calabrese, V, Cornelius, C, Rizzarelli, E, Owen, J. B, Dinkova-kostova, A. T, \& Butterfield, D. A. Nitric Oxide in Cell Survival: A Janus Molecule. Antioxidants \& Redox Signaling (2009). , 11(11)

[28] Spuch, C, Ortolano, S, \& Navarro, C. New Insights in the Amyloid-Beta Interaction withMitochondria. Journal of Aging Research Article ID 324968, 9 pages doi: 10.1155/2012/324968, 2012

[29] Walter, S, Letiembre, M, Liu, Y, Heine, H, Penke, B, Hao, W, Bode, B, \& Manietta, N. Jessica Walter J., Schulz-Schäffer W. and Faßbender K., Role of the Toll-Like Receptor 
4 in Neuroinflammation in Alzheimer's Disease. Cell Physiol Biochem (2007). , 20, 947-956.

[30] Medeiros, R, Prediger, R. D. S, Passos, G. F, Pandolfo, P, Duarte, F. S, Franco, J. L, Dafre, A. L, Giunta, G. D, Figueiredo, C. P, Takahashi, R. N, Campos, M. M, \& Calixto, J. B. Connecting TNF- $\beta$ Signaling Pathways to iNOS Expression in a Mouse Model of Alzheimer's Disease: Relevance for the Behavioral and Synaptic Deficits Induced by Amyloid $\beta$ Protein. Neurobiology of Disease, The Journal of Neuroscience, May 16, (2007). , 27(20), 5394-5404.

[31] Chandler, D, Woldu, A, Rahmadi, A, Shanmugam, K, Steiner, N, Wright, E, Benaventegarci, a O, Schulz, O, Castillo, J, \& Munch, G. Effects of plant-derived polyphenols on TNF- $\alpha$ and nitric oxide production induced by advanced glycation endproducts. Mol. Nutr. Food Res. (2010). SS150, 141.

[32] Wan, J, Fu, A. K. Y, \& Ip, F. C. F. Ho-Keung Ng, Hugon J., Page G., Wang J.H., KwokOn Lai, Wu Z., and Ip N.Y., Tyk2/STAT3 Signaling Mediates $\beta$-Amyloid-Induced Neuronal Cell Death: Implications in Alzheimer's Disease. Neurobiology of Disease The Journal of Neuroscience, May 19, (2010). , 30(20), 6873-6881.

[33] Wojtera, M, Sobów, T, Kloszewska, I, Liberski, P. P, Brown, D. R, \& Sikorska, B. Expression of immunohistochemical markers on microglia in Creutzfeldt-Jakob disease and Alzheimer's disease: morphometric study and review of the literature. Folia Neuropathol (2012). , 50(1), 74-84.

[34] Juan, E, Tichauer, J. E, \& Bernhardi, R. Transforming Growth Factor- $\beta$ Stimulates $\beta$ Amyloid Uptake by Microglia Through Smad3-Dependent Mechanisms. Journal of Neuroscience Research (2012). , 90, 1970-1980.

[35] Uribe, V, Wong, B.K.Y, Graham, R.K, Cusack, C.L, Skotte, N.H, Pouladi, M.A, Xie, Y, Feinberg, K, Ou, Y, Ouyang, Y, \& Deng, . ., Rescue from excitotoxicity and axonal degeneration accompanied by age-dependent behavioral and neuroanatomical alterations in caspase-6-deficient mice. Human Molecular Genetics, 2012; Vol. 21, No. 9 1954-1967

[36] Lee, J-H, \& Cheon, . ., Evidence of early involvement of apoptosis inducing factorinduced neuronal death in Alzheimer brain. Anatomy and Cell Biolog.2012; pISSN 2093-3665 eISSN 2093-3673. http://dx.doi.org/10.5115/acb.2012.45.1.26

[37] Zeng, L, Li, T, Xu, D. C, Liu, J, Mao, G, Cui, M. Z, Fu, X, \& Xu, X. Death Receptor 6 Induces Apoptosis Not through Type I or Type II Pathways, but via a Unique Mitochondria-dependent Pathway by Interacting with Bax Protein. J Biol Chem. 2012 Aug 17; Epub (2012). Jul 3., 287(34), 29125-33.

[38] Miranda, S, Opazo, C, \& Larrondo, L. F. MunÄ oz F.J., Ruiz F., Leighton F., Inestrosa N.C., The role of oxidative stress in the toxicity induced by amyloid b-peptide in Alzheimer's disease. Progress in Neurobiology (2000). , 62, 633-648. 
[39] Davenport, C. M, Sevastou, I. G, Hooper, C, Pocock, J. M, \& Inhibiting, P. Pathways in microglia attenuates microglial evoked neurotoxicity following exposure to Alzheimer peptides. Journal of Neurochemistry (2010). , 112, 552-563.

[40] Vargas, T, Ugalde, C, Spuch, C, Antequera, D, Moránc, M. J, Martín, M. A, Ferrer, I, Bermejo-pareja, F, \& Carro, E. A $\beta$ accumulation in choroid plexus is associated with mitochondrial-induced apoptosis. Neurobiology of Aging (2010). , 31, 1569-1581.

[41] Kudo, W, Lee, H-P, Smith, M. A, Zhu, X, Matsuyama, S, \& Lee, H-g. Inhibition of Bax protects neuronal cells from oligomeric $\mathrm{A} \beta$ neurotoxicity. Cell Death and Disease (2012). e309; doi:10.1038/cddis.2012.43

[42] Huang, T-C, \& Lu, K-T. Peter Wo Y-Y., Wu Y-J., Yang Y-L., Resveratrol Protects Rats from A $\beta$-induced Neurotoxicity by the Reduction of iNOS Expression and Lipid Peroxidation. PLoS ONE December (2011). e 29102, 6(12)

[43] XiaxieHai-Taowang, Chun-Lili, Xu-Hong Gao, Jin-Landing, Hai-Huazhao and YongLil, Ginsenoside Rb1 protects PC12 cells against $\beta$-amyloid-induced cell injury. Molecular Medicine REPORTS (2010). , 3, 635-639.

[44] Troy, C. M, \& Salvesen, G. S. Caspases On The Brain. Journal of Neuroscience Research (2002). , 69, 145-150.

[45] Chonga, Z. Z, Lia, F, \& Maiese, K. Stress in the brain: novel cellular mechanisms of injury linked to Alzheimer's disease. Brain Research Reviews (2005). , 49, 1-21. 
